\title{
Do weak magnetic fields prevent hydrogen from accreting onto metal-line white dwarf stars? ${ }^{\star}$
}

\author{
S. Friedrich ${ }^{1}$, S. Jordan ${ }^{2,3}$, and D. Koester ${ }^{4}$ \\ 1 Max-Planck-Institut für extraterrestrische Physik, Giessenbachstr., 85748 Garching, Germany \\ e-mail: sfriedrich@mpe.mpg.de \\ 2 Institut für Astronomie und Astrophysik, Sand 1, 72076 Tübingen, Germany \\ 3 Astronomisches Rechen-Institut, Mönchhofstr. 12-14, 69221 Heidelberg, Germany \\ e-mail: jordan@ari.uni-heidelberg.de \\ ${ }^{4}$ Institut für Theoretische Physik und Astrophysik, Universität Kiel, 24098 Kiel, Germany \\ e-mail: koester@astrophysik.uni-kiel.de
}

Received 29 April 2004 / Accepted 31 May 2004

\begin{abstract}
The widely accepted assumption is that metals detected in the spectra of a few cool helium-rich white dwarfs cannot be of primordial origin and therefore must be accreted from the interstellar medium. However, the observed abundances of hydrogen are much too low to be compatible with the high accretion rates inferred from metal accretion. Hydrogen accretion is therefore suppressed compared to metal accretion. The hypothesis most widely discussed as cause for this "hydrogen screening" is the propeller mechanism: Metals are accreted in the form of grains onto a slowly rotating, weakly magnetized white dwarf, whereas ionized hydrogen is repelled at the Alfvén radius. We have obtained circular polarization spectra of the helium-rich white dwarfs GD 40 (WD0300-013) and L745-46A (WD0738-172) - which both show strong metal lines as well as hydrogen in order to search for signatures of a weak magnetic field. The magnetic field strengths necessary for the propeller mechanism to work in these stars are at least $144000 \mathrm{G}$ and $3000 \mathrm{G}$, respectively. Whereas L745-46A might have a magnetic field of about $-6900 \mathrm{G}$ no magnetic field could be found with an upper limit for the field strength of $4000 \mathrm{G}$ (with 99\% confidence) for GD 40.
\end{abstract}

Key words. stars: individual: GD 40 - stars: individual: L745-46A - stars: white dwarfs - stars: magnetic fields

\section{Introduction}

Some of the helium-rich white dwarfs at the cool end of the white dwarf sequence show evidence for metal lines in their spectra. Since radiation forces are not strong enough to compete with gravity for temperatures below $40000 \mathrm{~K}$ it is expected that metals sink down on time scales which are short compared to the cooling age. If nevertheless metals are observed in the atmospheres, as in the DZ and related spectral types, they must have come from outside the star. The most popular mechanism for this is accretion from interstellar matter.

Based on earlier studies (Koester 1976; Wesemael 1979; Vauclair et al. 1979) concerning accretion processes onto cool white dwarfs, Dupuis et al. (1992, 1993a,b) developed a theoretical model which predicts element abundances in cool white dwarf atmospheres under the assumption that elements are accreted in solar element proportions from the interstellar medium. They followed the suggestion of Wesemael (1979) that due to the patchy structure of the interstellar medium a white dwarf may experience highly variable accretion rates during its life. Most of the time (about $5 \times 10^{7}$ years assumed in the strongly idealized scenario) the white dwarf will accrete

^ Based on observations collected at the European Southern Observatory, Paranal, Chile (ESO Programme 66.D-0541). at very low rates $\left(5 \times 10^{-20} M_{\odot} / y r\right)$ which lead to metal abundances in the photosphere well below the detection limit. Only during (or shortly after) encounters with denser patches in the interstellar medium (typical density 10 particles $\mathrm{cm}^{-3}$ and temperature $100 \mathrm{~K}$ ), metals are accreted with rates high enough to lead to detectable features in the spectra.

Whereas this scenario can account for the observed metal abundances in helium-rich white dwarfs, the observed abundances of hydrogen are much too low to be compatible with the accretion rates inferred from metal accretion (Dupuis et al. 1993b). In a recent study Wolff et al. (2002) analyzed the UV spectra of ten DZ and DBZ stars which form a homogenous data set and concluded that the observed hydrogen abundance is more than two orders of magnitude to low. Furthermore DZ white dwarfs are not found in or close to known dense clouds (Aannestad et al. 1993). One possible explanation is that hydrogen accretion is reduced compared to metal accretion. The mechanism most widely discussed as reason for this "hydrogen screening" is the propeller mechanism of Illarionov \& Sunyaev (1975).

\section{The propeller mechanism}

The propeller mechanism was originally developed for accretion onto neutron stars (Illarionov \& Sunyaev 1975) and 
adopted to white dwarfs by Wesemael \& Truran (1982). Wesemael \& Truran proposed that metals are accreted in the form of grains onto a slowly rotating, weakly magnetized white dwarf, whereas ionized hydrogen is repelled at the Alfvén radius. Figure 1 in Wesemael \& Truran shows the domain of allowed Alfvén radii as a function of luminosity (or temperature) of the central star. The domain is restricted to higher temperatures by setting the dust evaporation time scale at the Alfvén radius equal to the free-fall time from the accretion radius, to lower temperatures by equating the ionization radius to the Alfvén radius. For typical white dwarfs $\left(M=0.6 M_{\odot}\right)$ and accretion parameters $\left(\dot{M}=10^{-15} M_{\odot} / \mathrm{yr}, v=50 \mathrm{~km} \mathrm{~s}^{-1}\right)$ this region is roughly between $T_{\text {eff }}=7500 \mathrm{~K}$ and $T_{\text {eff }}=$ $15000 \mathrm{~K}$. The lower boundary can be bypassed if accretion from a warm ISM phase with temperature $T \approx 10000 \mathrm{~K}$ and density $n \approx 1 \mathrm{~cm}^{-3}$ is assumed. In such a phase, the hydrogen would be ionized, but the metals may still be partially locked up in grains (Alcock \& Illarionov 1980). Given the effective temperature of a star the minimum Alfvén radius, which in turn corresponds to a minimum magnetic field strength necessary to let the propeller mechanism work, can be calculated.

To our knowledge it has never been tested whether in helium-rich metal-line white dwarfs the necessary conditions for rotation, magnetic field, and strength of hydrogen-ionizing radiation are indeed fulfilled to make this mechanism operate over the entire range of $T_{\text {eff }}$ from $7500 \mathrm{~K}$ to $15000 \mathrm{~K}$.

\section{Observation and data reduction}

With effective temperatures of $15150 \mathrm{~K}$ and $7500 \mathrm{~K}$, respectively, GD 40 (WD0300-013, $V=15.56$ ) and L745-46A (WD0738-172, $V=13.04$ ) mark the upper and lower boundary of the temperature regime of the propeller mechanism. Both stars are well studied and atmospheric parameters as well as element abundances are known with sufficient accuracy. Based on the accretion-diffusion model of Dupuis et al. (1993b) the hydrogen accretion rates necessary to provide the observed hydrogen abundances are about three or four orders of magnitude lower than the average accretion rate which is assumed to account for the observed metal abundances (Friedrich et al. 1999; Koester \& Wolff 2000). Therefore, hydrogen accretion must be efficiently reduced even onto L745-46A which shows one of the highest hydrogen abundances observed in cool helium-rich white dwarfs.

For the above reasons both stars are ideal candidates to search for magnetic fields driving the propeller mechanism. The VLT with FORS1 and PMOS offers for the first time the possibility to obtain circular polarization spectra with high enough signal-to-noise ratios at acceptable exposure times to search for these magnetic fields as it has been demonstrated by Aznar Cuadrado et al. (2004) for white dwarfs.

Flux and circular polarization spectra were obtained with the VLT UT1 and PMOS in December 2000 in service mode. Grism 600R (dispersion $1 \AA /$ pix) was used with order sorting filter GG435 resulting in a spectral range of about 5300-7350 A. Several individual exposures were taken for each star. After each exposure the retarder plate was rotated by 90 degrees in order to account for improper flat field corrections, instrumental polarizations and depolarization. The resulting total exposure times for GD 40 and L745-46A were $3.3 \mathrm{~h}$ and $2.7 \mathrm{~h}$. The signal-to-noise ratio in the vicinity of $\mathrm{H} \alpha$ and the helium lines of GD 40 amounts to 190 and 260, respectively, and to 280 for $\mathrm{H} \alpha$ of L745-46A. Data reduction was done with standard IRAF routines. Circular polarization was first calculated for each individual retarder position from the difference of the double spectrum, produced by the Wollaston prism, divided by the sum of the double spectrum. Polarization spectra, corrected for instrumental influences, were then derived from the difference of two consecutive circular polarization spectra with different quarter wave plate positions. Subsequently these polarization spectra were averaged to get a mean circular polarization spectrum. The flux spectrum is given by the sum of both spectra. All individual flux spectra were averaged to get a mean flux spectrum.

In addition a star in the field of view of L745-46A, which we assume to be unpolarized, was observed. It served as a supplementary check for instrumental introduced polarization. The mean circular polarization in a spectral range between $5500 \AA$ and $6800 \AA$ amounts to $0.01 \%, 0.02 \%$, and $0.03 \%$ for the field star, GD 40, and L745-46A, respectively, one order of magnitude lower than the expected values for kilogauss magnetic fields.

\section{Determination of magnetic fields}

\subsection{Weak-field approximation}

According to the theory of line formation in a weak magnetic field the splitting of a spectral line is proportional to the mean longitudinal field $B_{1}$, i.e. the component of the magnetic field along the line of site averaged over the visible stellar surface. Provided that the Zeeman splitting of a spectral line is small compared to intrinsic (thermal and pressure) broadening (e.g., Angel \& Landstreet 1970) the amount of polarization can be determined by the weak-field approximation, which is valid even in the presence of instrumental broadening, but is not generally correct in the case of rotationally broadening (Landstreet 1982):

$\frac{V}{I}=-g_{\text {eff }} \frac{e}{4 \pi m_{\mathrm{e}} c^{2}} \lambda^{2} \frac{\mathrm{d} I}{I_{\lambda} \mathrm{d} \lambda} B_{1}$

with $e / 4 \pi m_{\mathrm{e}} c^{2} \approx 4.67 \times 10^{-13} \AA^{-1} \mathrm{G}^{-1}$, and $g_{\text {eff }}$ the effective Landé factor which equals 1 for hydrogen Balmer lines (e.g., Bagnulo et al. 2002, and citations therein). For the $\mathrm{He} \mathrm{I}$ line at $5875 \AA g_{\text {eff }}=1.16$ (Leone et al. 2000).

In our case the weak-field approximation is justified because intrinsic broadening is much larger than the separation of line components due to Zeeman splitting which is of the order of $0.1 \AA$ for field strengths of $10 \mathrm{kG}$. For higher field strengths which are called for by the propeller mechanism for GD 40 the intrinsic broadening is smaller than the magnetic broadening and the weak-field approximation reduces to a order-ofmagnitude estimation (Landstreet 1982).

Due to the lower signal-to-noise ratio at the $\mathrm{H} \alpha$ line of GD 40 the synthetic flux spectrum $I(\lambda)$ from Sect. 5.1.1 was 
used to calculate $V / I$. For L745-46A the $S / N$-ratio was sufficiently high to use the observed flux spectrum. $\mathrm{d} I / \mathrm{d} \lambda$ is approximated by the average of $\left(I_{i+1}-I_{i}\right) /\left(\lambda_{i+1}-\lambda_{i}\right)$ and $\left(I_{i}-I_{i-1}\right) /\left(\lambda_{i}-\lambda_{i-1}\right)$ for a given wavelength $\lambda_{i}$.

\subsection{Fitting of the magnetic field}

In order to determine the mean longitudinal component of the magnetic field the observed circular polarization was compared to the predictions of the weak-field approximation in an interval of $\pm 20 \AA$ around $\mathrm{H} \alpha$ and the He I lines at $5875 \AA$ and $6678 \AA$. The best-fit for $B_{1}$, the only free parameter, was found by a $\chi^{2}$ minimization procedure. If no magnetic field were present, all deviations from zero would be due to noise. This can be expressed by the standard deviation over the respective intervals around the respective lines. If the best-fit magnetic field is close to zero the reduced $\chi^{2}$ should be close to unity. However, if a magnetic field is present some deviations from zero are not due to noise and the reduced $\chi^{2}$ is smaller than 1 . Following Press et al. (1986) the statistical error is determined from the rms deviation of the observed circular polarization from the best-fit model. The $1 \sigma(68.3 \%)$ confidence range for a degree of freedom of 1 is the interval of $B_{1}$ where the deviation from the minimum is $\Delta \chi^{2}=1$ (6.63 for the $99 \%$ confidence range). This is a purely statistical error and does not account for systematic errors. For details see Aznar Cuadrado et al. (2004).

\subsection{Magnetic field strengths for the propeller mechanism}

Minimum magnetic field strengths necessary for the propeller mechanism to work were calculated according to Wesemael $\&$ Truran (1982). The logarithm of the Alvén radius was estimated from their Fig. 1. Assuming a white dwarf mass of $0.6 M_{\odot}$, a radius of $0.013 R_{\odot}$, an accretion rate of $10^{-15} M_{\odot} / \mathrm{yr}$ the corresponding minimum magnetic field strengths from their Eq. (4a) are $144000_{-17000}^{+25000} \mathrm{G}$ and $3000 \pm 500 \mathrm{G}$ for GD 40 and L745-46A, respectively. The error accounts for a reading error of $1 \mathrm{~mm}$ in their Fig. 1. Further errors are introduced by the stellar radius which enters the formula with third power and the accretion rate. Lower radii and higher accretion rates would increase the minimum magnetic field strength.

\section{Results}

\subsection{GD 40}

\subsubsection{Hydrogen abundance}

The flux spectrum of GD 40 clearly shows an $\mathrm{H} \alpha$ absorption line (Fig. 1) which confirms the detection of this line by Greenstein \& Liebert (1990). In disagreement to these authors, who determined an equivalent width of $1 \AA$, we measured $0.2 \AA$ only. We attribute the difference in the equivalent widths to the much better quality of our spectra compared to those of Greenstein \& Liebert. As a consequence the hydrogen abundance turned out to be a factor of 10 below the value

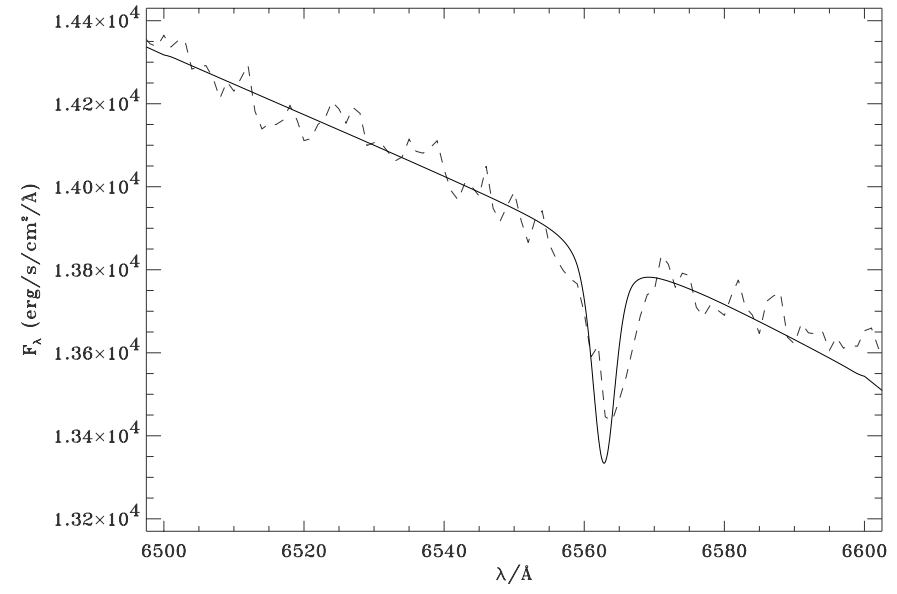

Fig. 1. Observed flux spectrum (dashed) of GD 40 at the position of $\mathrm{H} \alpha$ with a non-magnetic model spectrum (solid, $T_{\text {eff }}=15150$, $\log g=8$ ) over-plotted. The hydrogen abundance needed to reproduce the line profile of $\mathrm{H} \alpha$ amounts to $5 \times 10^{-7}$ relative to helium.

previously determined (Friedrich et al. 1999). In order to estimate the hydrogen abundance, we took the best fit, nonmagnetic model atmosphere with $T_{\text {eff }}=15150, \log g=8$ from Friedrich et al. (1999) and varied the hydrogen abundance while keeping all other element abundances and the atmospheric parameters fixed. This resulted in a new hydrogen abundance of $5 \times 10^{-7}$ relative to helium for GD 40 .

\subsubsection{Circular polarization}

The inspection of the individual polarization spectra of GD 40 did not show any obvious features or variations in the polarization which cannot be attributed to noise. The magnetic field strengths calculated with the theoretical weak-field approximation are below the $1 \sigma$ error. In addition different magnetic field orientations are found (indicated by positive and negative signs of $B_{1}$ ) for $\mathrm{H} \alpha$ and the two $\mathrm{He}_{\mathrm{I}}$ lines. For the $\mathrm{H} \alpha$ line the field strength and the respective $68.3 \% / 99 \%$ confidence ranges amount to $(2747 \pm 6892 / 15000) \mathrm{G}$, and for the two He I lines at $5875 \AA$ and $6678 \AA$ to $(-1683 \pm 1528 / 3936) \mathrm{G}$ and $(-716 \pm$ $1848 / 4758) \mathrm{G}$, respectively. A fit to all three lines results in a magnetic field strength of $1131 \mathrm{G}$ and a $99 \%$ confidence level of $\pm 2973 \mathrm{G}$. For comparison the derived field strength for the field star amounts to $-438 \pm 318 / 819 \mathrm{G}(68.3 \% / 99 \%$ confidence range). Thus we conclude that GD 40 does not posses a magnetic field with an upper limit of about $4000 \mathrm{G}$.

Figure 2 shows the observed circular polarization spectra of GD 40 at the $\mathrm{H} \alpha$ line and the two He I lines together with the respective polarization spectra with the magnetic field strengths predicted by the weak-field approximation and the maximum field strengths within the $68.3 \%$ and $99 \%$ confidence ranges.

\section{2. $L 745-46 A$}

Figure 3 shows the circular polarization at $\mathrm{H} \alpha$, which is the only visible line in the observed spectral range, together with the polarization spectrum for the best-fit magnetic field strength of $-6900 \mathrm{G}$. The $68.3 \%$ and $99 \%$ confidence ranges amount 



Fig. 2. Observed (gray) circular polarization spectrum of GD 40 together with the respective polarization spectra (bold) predicted by the weak-field approximation for field strengths of $2747 \mathrm{G}, 9639 \mathrm{G}$, and $17747 \mathrm{G}$ for $\mathrm{H} \alpha$ (top), $-1683 \mathrm{G},-3211 \mathrm{G}$, and $-5619 \mathrm{G}$ for the $\mathrm{He}_{\mathrm{I}}$ line at $5875 \AA$ (middle), and $-716 \mathrm{G},-2564 \mathrm{G}$, and $-5474 \mathrm{G}$ for the He I line at $6678 \AA$ (bottom). For each spectral line the first field strength denotes the best fit value, the second and third value the best fit value plus the $68.3 \%$ and $99 \%$ confidence range, respectively.

to $\pm 2100 \mathrm{G}$ and $\pm 5550 \mathrm{G}$. Again the polarization spectra with the maximum value from this field strength and the $68.3 \%$ and $99 \%$ confidence range are also plotted. Although the field strength clearly exceeds the statistical $1 \sigma$ error one has to be cautious, because the result solely depends on the vicinity of the $\mathrm{H} \alpha$ line. However, if this field could be confirmed, it would be strong enough to prevent hydrogen from accreting according to the prediction from the propeller mechanism.



Fig. 3. Observed (gray) circular polarization spectrum of L745-46A at $\mathrm{H} \alpha$ together with the polarization spectra (bold) determined by the weak-field approximation for field strengths of $-6900 \mathrm{G},-9000 \mathrm{G}$, and $-12450 \mathrm{G}$. The first field strength denotes the best fit value, the second and third value the the best fit value plus the $68.3 \%$ and $99 \%$ confidence range, respectively.

\section{Conclusion}

We have obtained high signal-to-noise circular polarization spectra of the helium-rich white dwarfs GD 40 and LP745-46A. With errors as low as $\pm 0.5 \%$ and $\pm 0.2 \%$ these spectra allow for the first time to search for the weak magnetic fields necessary to drive the so called propeller mechanism which is assumed to be responsible for the low observed hydrogen-to-metal ratios found in some cool helium-rich white dwarfs.

For L745-46A the predicted magnetic field strength from the weak field approximation amounts to $-6900 \mathrm{G}$ with a formal $1 \sigma$ error of $2100 \mathrm{G}$ and a $99 \%$ confidence range of $\pm 5550 \mathrm{G}$. This magnetic field strength exceeds the minimum field strength of $3000 \mathrm{G}$ necessary for the propeller mechanism to work. However, one should keep in mind, that this result is based on the $\mathrm{H} \alpha$ line only. If the detection is confirmed, this means for the first time an indication that magnetic fields may play a role in the accretion of hydrogen and metals onto cool helium-rich white dwarfs.

However, we could not detect signatures of a magnetic field in the circular polarization or flux spectrum of GD 40. This does not necessarily mean, that no magnetic field is present, because if we are looking on the magnetic equator of a magnetic dipole $\left(i=90^{\circ}\right)$, the components of the magnetic field along the line of sight completely cancel and no circular polarization can be detected. According to Wesemael \& Truran (1982) a field strength of about $144000 \mathrm{G}$ is needed for GD 40 to let the propeller mechanism work. If we now assume that we could detect a field strength of $4000 \mathrm{G}$ (derived upper limit field strength from the fit to all three lines with a confidence of 99\%) we could estimate an inclination angle of greater than $84^{\circ}$ for which the longitudinal field strength becomes to low to be detected (Brown et al. 1977). Therefore the chance to miss a magnetic field of $144000 \mathrm{G}$ is only about $6 \%$.

From our observations we must therefore conclude that at least for GD 40 other mechanisms than magnetic fields prevent hydrogen from accreting. Sion et al. (1990) argue that hydrogen deficient stars like GD 40, which do not lie close to any known 
interstellar cloud (Aannestad \& Sion 1985), have accreted, but not from interstellar clouds. They proposed that white dwarfs might experience accretion in different environments with some white dwarfs being accreting material from interstellar clouds and some from volatile-free asteroidal or dust material or chemically differentiated planetary material. Accretion of comets from an Oort-like cloud as proposed by Alcock et al. (1986), however, failed to explain the observed $\mathrm{Ca} / \mathrm{H}$ ratios and is ruled out by Zuckerman et al. (2003) as a source of metals at least for DAZ white dwarfs. In view of the suggestion that the DAZ phenomenon might be associated with the existence of a nearby unseen companion (Zuckerman \& Reid 1998), Zuckerman et al. (2003) also speculate about Jupitersize substellar companions of DA and DB white dwarfs, that might generate detectable metal-line absorption. One problem with this model is lifting material off the secondary. Another problem might be the lack of observed eclipses, which would be expected, if a substantial number of all DAs and DBs are orbited by such substellar companions with semi-major axis of a few solar radii, which are typical for red dwarf/white dwarf pairs. A dusty circumstellar disk might be also the reason for a hydrogen depleted environment as mentioned in Aannestad et al. (1993). The maintenance of such a disk over the long lifetime of a cool white dwarf, however, would be difficult to understand.

From the determination of hydrogen and metal abundances in helium-rich white dwarfs one must conclude that predominantly metals are accreted. However, the reasons for this selective accretion process are not yet really understood. It seems that also other mechanism than magnetic fields must be considered.

Acknowledgements. We thank the ESO staff on Cerro Paranal for observing GD 40 and L745-46A with the VLT in service mode. Work on magnetic white dwarfs in Kiel was supported by the DFG under KO-738/7-1. In Tübingen work is supported by the DLR under 50 OR 0201 .

\section{References}

Aannestad, P. A., Kenyon, S. J., Hammond, G. L., \& Sion, E. M. 1993, AJ, 105, 1033

Aannestad, P. A., \& Sion, E. M. 1985, AJ, 90, 832

Alcock, C., \& Illarionov, A. 1980, ApJ, 235, 534

Alcock, C., Fristrom, C. C., \& Siegelman, R. 1986, ApJ, 302, 462

Angel, J. R. P., \& Landstreet, J. D. 1970, ApJ, 160, L147

Aznar Cuadrado, R., Jordan, S., Napiwotzki, R., et al. 2004, in press [arXiv: astro-ph/0405308]

Bagnulo, S., Szeifert, T., Wade, G. A., Landstreet, J. D., \& Mathys, G. 2002, A\&A, 389, 191

Brown, D. N., Rich, A., Williams, W. L., \& Vauclair, G. 1977, ApJ, 218, 227

Dupuis, J., Fontaine, G., Pelletier, C., \& Wesemael, F. 1992, ApJS, 82, 505

Dupuis, J., Fontaine, G., Pelletier, C., \& Wesemael, F. 1993a, ApJS, 84,73

Dupuis, J., Fontaine, G., \& Wesemael, F. 1993b, ApJS, 87, 345

Friedrich, S., Koester, D., Heber, U., Jeffery, C. S., \& Reimers, D. 1999, A\&A, 350, 865

Greenstein, J. L., \& Liebert, J. W. 1990, ApJ, 360, 662

Illarionov, A. F., \& Sunyaev, R. A. 1975, A\&A, 39, 185

Koester, D. 1976, A\&A, 52, 415

Koester, D., \& Wolff, B. 2000, A\&A, 357, 587

Landstreet, J. D. 1982, ApJ, 258, 639

Leone, F., Catanzaro, G., \& Catalano, S. 2000, A\&A, 355, 315

Press, W. H., Flannery, B. P., \& Teukolsky, S. A. 1986, Numerical Recipes (Cambridge: Univ. Press)

Sion, E. M., Hammond, G. L., Wagner, R. M., \& Starrfield, S. G. 1990, ApJ, 362, 691

Vauclair, G., Vauclair, S., \& Greenstein, J. L. 1979, A\&A, 80, 79

Wesemael, F. 1979, A\&A, 72, 104

Wesemael, F., \& Truran, J. W. 1982, ApJ, 260, 807

Wolff, B., Koester, D., \& Liebert, J. 2002, A\&A, 385, 995

Zuckerman, B., \& Reid, I. N. 1998, ApJ, 505, L143

Zuckerman, B., Koester, D., Reid, I. N., \& Hünsch, M. 2003, ApJ, 596,477 